\title{
The Group of Quasisymmetric Homeomorphisms of the Circle and Quantization of the Universal Teichmüller Space ${ }^{\star}$
}

\author{
Armen G. SERGEEV \\ Steklov Mathematical Institute, 8 Gubkina Str., 119991 Moscow, Russia \\ E-mail: sergeev@mi.ras.ru
}

Received July 29, 2008, in final form February 05, 2009; Published online February 08, 2009

doi:10.3842/SIGMA.2009.015

\begin{abstract}
In the first part of the paper we describe the complex geometry of the universal Teichmüller space $\mathcal{T}$, which may be realized as an open subset in the complex Banach space of holomorphic quadratic differentials in the unit disc. The quotient $\mathcal{S}$ of the diffeomorphism group of the circle modulo Möbius transformations may be treated as a smooth part of $\mathcal{T}$. In the second part we consider the quantization of universal Teichmüller space $\mathcal{T}$. We explain first how to quantize the smooth part $\mathcal{S}$ by embedding it into a Hilbert-Schmidt Siegel disc. This quantization method, however, does not apply to the whole universal Teichmüller space $\mathcal{T}$, for its quantization we use an approach, due to Connes.
\end{abstract}

Key words: universal Teichmüller space; quasisymmetric homeomorphisms; Connes quantization

2000 Mathematics Subject Classification: 58E20; 53C28; 32L25

\section{Introduction}

The universal Teichmüller space $\mathcal{T}$, introduced by Ahlfors and Bers, plays a key role in the theory of quasiconformal maps and Riemann surfaces. It can be defined as the space of quasisymmetric homeomorphisms of the unit circle $S^{1}$ (i.e. homeomorphisms of $S^{1}$, extending to quasiconformal maps of the unit disc $\Delta$ ) modulo Möbius transformations. The space $\mathcal{T}$ has a natural complex structure, induced by its realization as an open subset in the complex Banach space $B_{2}(\Delta)$ of holomorphic quadratic differentials in the unit disc $\Delta$. The space $\mathcal{T}$ contains all classical Teichmüller spaces $T(G)$, where $G$ is a Fuchsian group, as complex submanifolds. The space $\mathcal{S}:=\operatorname{Diff}_{+}\left(S^{1}\right) / \operatorname{Möb}\left(S^{1}\right)$ of normalized diffeomorphisms of the circle may be considered as a "smooth" part of $\mathcal{T}$.

Our motivation to study $\mathcal{T}$ comes from the string theory. Physicists have noticed (cf. [15, 3]) that the space $\Omega_{d}:=C_{0}^{\infty}\left(S^{1}, \mathbb{R}^{d}\right)$ of smooth loops in the $d$-dimensional vector space $\mathbb{R}^{d}$ may be identified with the phase space of bosonic closed string theory. By looking at a natural symplectic form $\omega$ on $\Omega_{d}$, induced by the standard symplectic form (of type " $d p \wedge d q$ ") on the phase space, one sees that this form can be, in fact, extended to the Sobolev completion of $\Omega_{d}$, coinciding with the space $V_{d}:=H_{0}^{1 / 2}\left(S^{1}, \mathbb{R}^{d}\right)$ of half-differentiable vector-functions. Moreover, the latter space is the largest in the scale of Sobolev spaces $H_{0}^{s}\left(S^{1}, \mathbb{R}^{d}\right)$, on which $\omega$ is correctly defined. So the form $\omega$ itself chooses the "right" space to be defined on. From that point of view, it seems more natural to consider $V_{d}$ as the phase space of bosonic string theory, rather than $\Omega_{d}$. In this paper we set $d=1$ to simplify the formulas and study the space $V:=V_{1}=H_{0}^{1 / 2}\left(S^{1}, \mathbb{R}\right)$.

\footnotetext{
${ }^{\star}$ This paper is a contribution to the Special Issue on Kac-Moody Algebras and Applications. The full collection is available at http://www.emis.de/journals/SIGMA/Kac-Moody_algebras.html
} 
According to Nag-Sullivan [12], there is a natural group, attached to the space $V=H_{0}^{1 / 2}\left(S^{1}, \mathbb{R}\right)$, and this is precisely the group $\mathrm{QS}\left(S^{1}\right)$ of quasisymmetric homeomorphisms of the circle. Again one can say that the space $V$ itself chooses the "right" group to be acted on. The group $\mathrm{QS}\left(S^{1}\right)$ acts on $V$ by reparametrization of loops and this action is symplectic with respect to the form $\omega$. The universal Teichmüller space $\mathcal{T}=\mathrm{QS}\left(S^{1}\right) / \operatorname{Möb}\left(S^{1}\right)$ can be identified by this action with a space of complex structures on $V$, compatible with $\omega$.

The second half of the paper is devoted to the quantization of the universal Teichmüller space $\mathcal{T}$. We start from the Dirac quantization of the smooth part $\mathcal{S}=\operatorname{Diff}_{+}\left(S^{1}\right) / \operatorname{Möb}\left(S^{1}\right)$. This is achieved by embedding of $\mathcal{S}$ into the Hilbert-Schmidt Siegel disc $\mathcal{D}_{\text {HS }}$. Under this embedding the diffeomorphism group Diff $\left(S^{1}\right)$ is realized as a subgroup of the Hilbert-Schmidt symplectic group $\operatorname{Sp}_{\mathrm{HS}}(V)$, acting on the Siegel disc by operator fractional-linear transformations. There is a holomorphic Fock bundle $\mathcal{F}$ over $\mathcal{D}_{\mathrm{HS}}$, provided with a projective action of $\operatorname{Sp}_{\mathrm{HS}}(V)$, covering its action on $\mathcal{D}_{\text {HS }}$. The infinitesimal version of this action is a projective representation of the Hilbert-Schmidt symplectic Lie algebra $\operatorname{sp}_{\mathrm{HS}}(V)$ in a fibre $F_{0}$ of the Fock bundle $\mathcal{F}$. This defines the Dirac quantization of the Siegel disc $\mathcal{D}_{\text {HS }}$. Its restriction to $\mathcal{S}$ gives a projective representation of the Lie algebra $\operatorname{Vect}\left(S^{1}\right)$ of the group $\operatorname{Diff}_{+}\left(S^{1}\right)$ in the Fock space $F_{0}$, which defines the Dirac quantization of the space $\mathcal{S}$.

However, the described quantization procedure does not apply to the whole universal Teichmüller space $\mathcal{T}$. By this reason we choose another approach to this problem, based on Connes quantization. (We are grateful to Alain Connes for drawing our attention to this approach, presented in [5].) Briefly, the idea is the following. The $\mathrm{QS}\left(S^{1}\right)$-action on $\mathcal{T}$, mentioned above, cannot be differentiated in classical sense (in particular, there is no Lie algebra, associated to $\left.\mathrm{QS}\left(S^{1}\right)\right)$. However, one can define a quantized infinitesimal version of this action by associating with any quasisymmetric homeomorphism $f \in \mathrm{QS}\left(S^{1}\right)$ a quantum differential $d^{q} f$, being an integral operator on $V$ with kernel, given essentially by the finite-difference derivative of $f$. In these terms the quantization of $\mathcal{T}$ is given by a representation of the algebra of derivations of $V$, generated by quantum differentials $d^{q} f$, in the Fock space $F_{0}$.

\section{Universal Teichmüller space}

\section{Group of quasisymmetric homeomorphisms of $S^{1}$}

\subsection{Definition of quasisymmetric homeomorphisms}

Definition 1. A homeomorphism $h: S^{1} \rightarrow S^{1}$ is called quasisymmetric if it can be extended to a quasiconformal homeomorphism $w$ of the unit disc $\Delta$.

Recall that a homeomorphism $w: \Delta \rightarrow w(\Delta)$, having locally $L^{1}$-integrable derivatives (in generalized sense), is called quasiconformal if there exists a measurable complex-valued function

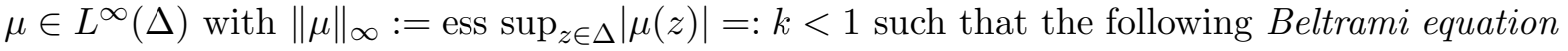

$$
w_{\bar{z}}=\mu w_{z}
$$

holds for almost all $z \in \Delta$. The function $\mu$ is called a Beltrami differential or Beltrami potential of $w$ and the constant $k$ is often indicated in the name of the $k$-quasiconformal maps.

In the case when $k=0$ the homeomorphism $w$, satisfying (1), coincides with a conformal map from $D$ onto $w(D)$. For a diffeomorphism $w$ its quasiconformality means that $w$ transforms infinitesimal circles into infinitesimal ellipses, whose eccentricities (the ratio of the large axis to the small one) are bounded by a common constant $K<\infty$, related to the above constant $k=\|\mu\|_{\infty}$ by the formula

$$
K=\frac{1+k}{1-k} .
$$


The least possible constant $K$ is called the maximal dilatation of $w$ and is also sometimes indicated in the name of $K$-quasiconformal maps.

The inverse of a quasiconformal map is again quasiconformal and the same is true for the composition of quasiconformal maps. This implies that orientation-preserving quasisymmetric homeomorphisms of $S^{1}$ form a group of quasisymmetric homeomorphisms of the circle $\mathrm{QS}\left(S^{1}\right)$ with respect to composition.

Any orientation-preserving diffeomorphism $h \in \operatorname{Diff}_{+}\left(S^{1}\right)$ extends to a diffeomorphism of the closed unit disc $\bar{\Delta}$, which is evidently quasiconformal, according to the above criterion. So $\operatorname{Diff}_{+}\left(S^{1}\right) \subset \mathrm{QS}\left(S^{1}\right)$, and we have the following chain of embeddings

$$
\operatorname{Möb}\left(S^{1}\right) \subset \operatorname{Diff}_{+}\left(S^{1}\right) \subset \operatorname{QS}\left(S^{1}\right) \subset \operatorname{Homeo}_{+}\left(S^{1}\right) .
$$

Here, $\operatorname{Möb}\left(S^{1}\right)$ denotes the Möbius group of fractional-linear automorphisms of the unit disc $\Delta$, restricted to $S^{1}$.

\subsection{Beurling-Ahlfors criterion}

There is an intrinsic description of quasisymmetric homeomorphisms of $S^{1}$ in terms of cross ratios. Recall that the cross ratio of four different points $z_{1}, z_{2}, z_{3}, z_{4}$ on the complex plane is given by the quantity

$$
\rho=\rho\left(z_{1}, z_{2}, z_{3}, z_{4}\right):=\frac{z_{4}-z_{1}}{z_{4}-z_{2}}: \frac{z_{3}-z_{1}}{z_{3}-z_{2}} .
$$

The equality of two cross ratios $\rho\left(z_{1}, z_{2}, z_{3}, z_{4}\right)=\rho\left(\zeta_{1}, \zeta_{2}, \zeta_{3}, \zeta_{4}\right)$ is a necessary and sufficient condition for the existence of a fractional-linear map of the complex plane, transforming the quadruple $z_{1}, z_{2}, z_{3}, z_{4}$ into the quadruple $\zeta_{1}, \zeta_{2}, \zeta_{3}, \zeta_{4}$. In the case of quasiconformal maps the cross ratios of quadruples may change but in a controlled way. This property, reformulated in the right way for orientation-preserving homeomorphisms of $S^{1}$, yields a criterion of quasisymmetricity, due to Ahlfors and Beurling.

The required property reads as follows: for an orientation-preserving homeomorphism $h$ : $S^{1} \rightarrow S^{1}$ it should exist a constant $0<\epsilon<1$ such that the following inequality holds

$$
\frac{1}{2}(1-\epsilon) \leq \rho\left(h\left(z_{1}\right), h\left(z_{2}\right), h\left(z_{3}\right), h\left(z_{4}\right)\right) \leq \frac{1}{2}(1+\epsilon)
$$

for any quadruple $z_{1}, z_{2}, z_{3}, z_{4} \in S^{1}$ with cross ratio $\rho\left(z_{1}, z_{2}, z_{3}, z_{4}\right)=\frac{1}{2}$.

Theorem 1 (Beurling-Ahlfors, cf. [1,9]). Suppose that $h: S^{1} \rightarrow S^{1}$ is an orientationpreserving homeomorphism of $S^{1}$. Then it can be extended to a quasiconformal homeomorphism $w: \Delta \rightarrow \Delta$ if and only if it satisfies condition (2).

Douady and Earle (cf. [6]) have found an explicit extension operator E, assigning to a quasisymmetric homeomorphism $h$ its extension to a quasiconformal homeomorphism $w$ of $\Delta$, which is conformally invariant in the sense that $g(w \circ h)=w \circ g(h)$ for any fractional-linear automorphism of $\Delta$.

Though quasisymmetric homeomorphisms of $S^{1}$, in general, are not smooth, they enjoy certain Hölder continuity, provided by the following

Theorem 2 (Mori, cf. [1]). Let $w: \Delta \rightarrow \Delta$ be a K-quasiconformal homeomorphism of the unit disc onto itself, normalized by the condition: $w(0)=0$. Then the following sharp estimate

$$
\left|w\left(z_{1}\right)-w\left(z_{2}\right)\right|<16\left|z_{1}-z_{2}\right|^{1 / K}
$$

holds for any $z_{1} \neq z_{2} \in \Delta$. In other words, the homeomorphism $w$ satisfies the Hölder condition of order $1 / K$ in the disc $\Delta$. 


\section{Universal Teichmüller space}

\subsection{Definition of universal Teichmüller space}

Definition 2. The quotient space

$$
\mathcal{T}:=\operatorname{QS}\left(S^{1}\right) / \operatorname{Möb}\left(S^{1}\right)
$$

is called the universal Teichmüller space. It can be identified with the space of normalized quasisymmetric homeomorphisms of $S^{1}$, fixing the points \pm 1 and $-i$.

As we have pointed out earlier, there is an inclusion

$$
\operatorname{Diff}_{+}\left(S^{1}\right) / \operatorname{Möb}\left(S^{1}\right) \hookrightarrow \mathcal{T}=\mathrm{QS}\left(S^{1}\right) / \operatorname{Möb}\left(S^{1}\right) .
$$

We consider the homogeneous space

$$
\mathcal{S}:=\operatorname{Diff}_{+}\left(S^{1}\right) / \operatorname{Möb}\left(S^{1}\right)
$$

as a "smooth" part of $\mathcal{T}$.

The space $\mathcal{T}$ can be provided with the Teichmüller distance function, defined by

$$
\operatorname{dist}(g, h)=\frac{1}{2} \log K\left(h \circ g^{-1}\right)
$$

for any quasisymmetric homeomorphisms $g, h \in \mathcal{T}$, extended to quasiconformal homeomorphisms of the disc $\Delta$. Here, $K\left(h \circ g^{-1}\right)$ denotes the maximal dilatation of the quasiconformal map $h \circ g^{-1}$. This definition does not depend on the extensions of $g, h$ to $\Delta$ and defines a metric on $\mathcal{T}$. The universal Teichmüller space is a complete connected contractible metric space with respect to the introduced distance function (cf. [9]). Unfortunately, this metric is not compatible with the group structure on $\mathcal{T}$, given by composition of quasisymmetric homeomorphisms (cf. [9, Theorem 3.3]).

The term "universal" in the name of the universal Teichmüller space is due to the fact that $\mathcal{T}$ contains, as complex submanifolds, all classical Teichmüller spaces $T(G)$, where $G$ is a Fuchsian group (cf. [10]). If a Riemann surface $X$ is uniformized by the unit disc $\Delta$, so that $X=\Delta / G$, then the corresponding Techmüller space $T(G)$ may be identified with the quotient

$$
T(G)=\operatorname{QS}\left(S^{1}\right)^{G} / \operatorname{Möb}\left(S^{1}\right),
$$

where $\operatorname{QS}\left(S^{1}\right)^{G}$ is the subset of $G$-invariant quasisymmetric homeomorphisms in $\operatorname{QS}\left(S^{1}\right)$. The universal Teichmüller space $\mathcal{T}$ itself corresponds to the Fuchsian group $G=\{1\}$.

Since quasisymmetric homeomorphisms of $S^{1}$ are defined in terms of quasiconformal maps of $\Delta$, i.e. in terms of solutions of Beltrami equation in $\Delta$, one can expect that there is a definition of $\mathcal{T}$ directly in terms of Beltrami differentials. Denote by $B(\Delta)$ the set of Beltrami differentials in the unit disc $\Delta$. It follows from above that it can be identified (as a set) with the unit ball in the complex Banach space $L^{\infty}(\Delta)$.

Given a Beltrami differential $\mu \in B(\Delta)$, we can extend it to a Beltrami differential $\check{\mu}$ on the extended complex plane $\overline{\mathbb{C}}$ by setting $\check{\mu}$ equal to zero outside the unit disc $\Delta$. Then, applying the existence theorem for quasiconformal maps on the extended complex plane $\overline{\mathbb{C}}$ (cf. [1]), we get a normalized quasiconformal homeomorphism $w^{\mu}$, satisfying Beltrami equation (1) on $\overline{\mathbb{C}}$ with potential $\check{\mu}$. This homeomorphism is conformal on the exterior $\Delta_{-}$of the closed unit disc $\bar{\Delta}$ on $\overline{\mathbb{C}}$ and fixes the points $\pm 1,-i$. The image $\Delta^{\mu}:=w^{\mu}(\Delta)$ of $\Delta$ under the quasiconformal map $w^{\mu}$ is called a quasidisc. We associate with Beltrami differential $\mu \in B(\Delta)$ the normalized quasidisc $\Delta^{\mu}$. Introduce an equivalence relation between Beltrami differentials in $\Delta$ by saying 
that two Beltrami differentials $\mu$ and $\nu$ are equivalent if $\left.\left.w^{\mu}\right|_{\Delta_{-}} \equiv w^{\nu}\right|_{\Delta_{-}}$. Then the universal Teichmüller space $\mathcal{T}$ will coincide with the quotient

$$
\mathcal{T}=B(\Delta) / \sim
$$

of the space $B(\Delta)$ of Beltrami differentials modulo introduced equivalence relation. In other words, it coincides with the space of normalized quasidiscs in $\overline{\mathbb{C}}$.

\subsection{Complex structure of the universal Teichmüller space}

We introduce a complex structure on the universal Teichmüller space $\mathcal{T}$, using its embedding into the space of quadratic differentials.

Given an arbitrary point $[\mu]$ of $\mathcal{T}$, represented by a normalized quasidisc $w^{\mu}(\Delta)$, consider a map

$$
\mu \longmapsto S\left(\left.w^{\mu}\right|_{\Delta_{-}}\right),
$$

assigning to a Beltrami differential $\mu \in[\mu]$ the Schwarz derivative of the conformal map $w^{\mu}$ on $\Delta$. Due to the invariance of Schwarzian under Möbius transformations, the image of $\mu$ under the above map depends only on the class $[\mu]$ of $\mu$ in $\mathcal{T}$. Moreover, it is a holomorphic quadratic differentials in $\Delta_{-}$. The latter fact follows from the transformation properties of Beltrami differentials, prescribed by Beltrami equation (according to (1), Beltrami differential behaves as a $(-1,1)$-differential with respect to conformal changes of variable). Composing the above map with a fractional-linear biholomorphism of $\Delta_{-}$onto the unit disc $\Delta$, we obtain a map

$$
\Psi: \mathcal{T} \longrightarrow B_{2}(\Delta), \quad[\mu] \longmapsto \psi(\mu),
$$

associating a holomorphic quadratic differential $\psi(\mu)$ in $\Delta$ with a point $[\mu]$ of the universal Teichmüller space $\mathcal{T}$.

The space $B_{2}(\Delta)$ of holomorphic quadratic differentials in $\Delta$ is a complex Banach space, provided with a natural hyperbolic norm, given by

$$
\|\psi\|_{2}:=\sup _{z \in \Delta}\left(1-|z|^{2}\right)^{2}|\psi(z)|
$$

for a quadratic differential $\psi$. It can be proved (cf. [9]) that $\|\psi[\mu]\|_{2} \leq 6$ for any Beltrami differential $\mu \in B(\Delta)$.

The constructed map $\Psi: \mathcal{T} \rightarrow B_{2}(\Delta)$, called a Bers embedding, is a homeomorphism of $\mathcal{T}$ onto an open bounded connected contractible subset in $B_{2}(\Delta)$, containing the ball of radius $1 / 2$, centered at the origin (cf. [9]).

Using the constructed embedding, we can introduce a complex structure on the universal Teichmüller space $\mathcal{T}$ by pulling it back from the complex Banach space $B_{2}(\Delta)$. It provides $\mathcal{T}$ with the structure of a complex Banach manifold. (Note that the topology on $\mathcal{T}$, induced by the map $\Psi$, is equivalent to the one, determined by the Teichmüller distance function.)

Moreover, the composition of the natural projection

$$
B(\Delta) \longrightarrow \mathcal{T}=B(\Delta) / \sim
$$

with the constructed map $\Psi$ yields a holomorphic map

$$
F: B(\Delta) \longrightarrow B_{2}(\Delta)
$$

with respect to the natural complex structure on $B(\Delta)$ (cf. [10]). 


\section{QS-action on the Sobolev space of half-differentiable functions}

\section{Sobolev space of half-differentiable functions on $S^{1}$}

\subsection{Definition}

The Sobolev space of half-differentiable functions on $S^{1}$ is a Hilbert space $V:=H_{0}^{1 / 2}\left(S^{1}, \mathbb{R}\right)$, consisting of functions $f \in L^{2}\left(S^{1}, \mathbb{R}\right)$ with zero average over the circle, having generalized derivatives of order $1 / 2$ again in $L^{2}\left(S^{1}, \mathbb{R}\right)$. In terms of Fourier series, a function $f \in L^{2}\left(S^{1}, \mathbb{R}\right)$ with Fourier series

$$
f(z)=\sum_{k \neq 0} f_{k} z^{k}, \quad f_{k}=\bar{f}_{-k}, \quad z=e^{i \theta},
$$

belongs to $H_{0}^{1 / 2}\left(S^{1}, \mathbb{R}\right)$ if and only if it has a finite Sobolev norm of order $1 / 2$ :

$$
\|f\|_{1 / 2}^{2}=\sum_{k \neq 0}|k|\left|f_{k}\right|^{2}=2 \sum_{k>0} k\left|f_{k}\right|^{2}<\infty .
$$

The space $H_{0}^{1 / 2}\left(S^{1}, \mathbb{R}\right)$ is well known and widely used in classical function theory (cf. [18]). However, our motivation to employ this space comes from its relation to string theory (cf. below).

\subsection{Kähler structure}

A symplectic form on $V$ is given by a 2-form $\omega: V \times V \rightarrow \mathbb{R}$, defined in terms of Fourier coefficients of $\xi, \eta \in V$ by

$$
\omega(\xi, \eta)=2 \operatorname{Im} \sum_{k>0} k \xi_{k} \bar{\eta}_{k}
$$

Because of (3), this form is correctly defined on $V$. Moreover, $H_{0}^{1 / 2}\left(S^{1}, \mathbb{R}\right)$ is the largest Hilbert space in the scale of Sobolev spaces $H_{0}^{s}\left(S^{1}, \mathbb{R}\right), s \in \mathbb{R}$, on which this form is defined. It should be also underlined that the form $\omega$ is the only natural symplectic form on $V$ (we shall make this point clear in Section 5.1).

We return to our motivation for studying the space $V$. It is well known to physicists (cf., e.g., $[15,3])$ that the space $\Omega_{d}=C_{0}^{\infty}\left(S^{1}, \mathbb{R}^{d}\right)$ of smooth loops in the $d$-dimensional vector space $\mathbb{R}^{d}$ can be identified with the phase space of bosonic closed string theory. The space $\Omega_{d}$ has a natural symplectic form, which coincides with the image of the standard symplectic form (of type " $d p \wedge d q$ ") on the phase space of closed string theory under the above identification. This form, computed in terms of Fourier decompositions, coincides precisely with the form $\omega$, given by (4). As we have remarked, the latter form may be extended to the Sobolev space $V_{d}:=$ $H_{0}^{1 / 2}\left(S^{1}, \mathbb{R}^{d}\right)$ and this space is the largest in the scale $H_{0}^{s}\left(S^{1}, \mathbb{R}^{d}\right)$ of Sobolev spaces, on which $\omega$ is correctly defined. One can say that symplectic form $\omega$ "chooses" the Sobolev space $V_{d}$. This is in contrast to $\Omega_{d}$, which was taken for the phase space of string theory simply because it's easier to work with smooth loops. By this reason, we find it more natural to consider $V_{d}$ as the phase space of string theory, which motivates the study of $V_{d}$ in more detail. In our analysis we set $d=1$ for simplicity.

Apart from symplectic form, the Sobolev space $V$ has a complex structure $J^{0}$, which can be given in terms of Fourier decompositions by the formula

$$
\xi(z)=\sum_{k \neq 0} \xi_{k} z^{k} \longmapsto\left(J^{0} \xi\right)(z)=-i \sum_{k>0} \xi_{k} z^{k}+i \sum_{k<0} \xi_{k} z^{k}
$$


This complex structure is compatible with symplectic form $\omega$ and, in particular, defines a Kähler metric $g^{0}$ on $V$ by $g^{0}(\xi, \eta):=\omega\left(\xi, J^{0} \eta\right)$ or, in terms of Fourier decompositions,

$$
g^{0}(\xi, \eta)=2 \operatorname{Re} \sum_{k>0} k \xi_{k} \bar{\eta}_{k}
$$

In other words, $V$ has the structure of a Kähler Hilbert space.

The complexification $V^{\mathbb{C}}=H_{0}^{1 / 2}\left(S^{1}, \mathbb{C}\right)$ of $V$ is a complex Hilbert space and the Kähler metric $g^{0}$ on $V$ extends to a Hermitian inner product on $V^{\mathbb{C}}$, given by

$$
\langle\xi, \eta\rangle=\sum_{k \neq 0}|k| \xi_{k} \bar{\eta}_{k}
$$

We extend the symplectic form $\omega$ and complex structure operator $J^{0}$ complex linearly to $V^{\mathbb{C}}$.

The space $V^{\mathbb{C}}$ is decomposed into the direct sum of the form

$$
V^{\mathbb{C}}=W_{+} \oplus W_{-},
$$

where $W_{ \pm}$is the $(\mp i)$-eigenspace of the operator $J^{0} \in$ End $V^{\mathbb{C}}$. In other words,

$$
W_{+}=\left\{f \in V^{\mathbb{C}}: f(z)=\sum_{k>0} f_{k} z^{k}\right\}, \quad W_{-}=\bar{W}_{+}=\left\{f \in V^{\mathbb{C}}: f(z)=\sum_{k<0} f_{k} z^{k}\right\} .
$$

The subspaces $W_{ \pm}$are isotropic with respect to symplectic form $\omega$ and the splitting $V^{\mathbb{C}}=$ $W_{+} \oplus W_{-}$is an orthogonal direct sum with respect to the Hermitian inner product $\langle\cdot, \cdot\rangle$, given by (5).

\section{Grassmann realization of $\mathcal{T}$}

\subsection{QS-action on the Sobolev space}

Note that any homeomorphism $h$ of $S^{1}$, preserving the orientation, acts on $L_{0}^{2}\left(S^{1}, \mathbb{R}\right)$ by change of variable. In other words, there is an operator $T_{h}: L_{0}^{2}\left(S^{1}, \mathbb{R}\right) \rightarrow L_{0}^{2}\left(S^{1}, \mathbb{R}\right)$, acting by

$$
T_{h}(\xi):=\xi \circ h-\frac{1}{2 \pi} \int_{0}^{2 \pi} \xi(h(\theta)) d \theta .
$$

This operator has the following remarkable property.

Proposition 1 (Nag-Sullivan [12]). The operator $T_{h}$ acts on $V$, i.e. $T_{h}: V \rightarrow V$, if and only if $h \in \mathrm{QS}\left(S^{1}\right)$. Moreover, if $h$ extends to a $K$-quasiconformal homeomorphism of the unit disc $\Delta$, then the operator norm of $T_{h}$ does not exceed $\sqrt{K+K^{-1}}$, where $K=K(h)$ is the maximal dilatation of $h$.

Moreover, transformations $T_{h}$ with $h \in \mathrm{QS}\left(S^{1}\right)$ generate symplectic transformations of $V$.

Proposition 2 (Nag-Sullivan [12]). For any $h \in \mathrm{QS}\left(S^{1}\right)$ we have

$$
\omega\left(h^{*}(\xi), h^{*}(\eta)\right)=\omega(\xi, \eta)
$$

for all $\xi, \eta \in V$. Moreover, the complex-linear extension of QS-action to the complexification $V^{\mathbb{C}}$ preserves the holomorphic subspace $W_{+}$if and only if $h \in \operatorname{Möb}\left(S^{1}\right)$. In the latter case, $T_{h}$ acts as a unitary operator on $W_{+}$. 
We have pointed out in Section 4.2 that the Sobolev space $V$ is "chosen" by the symplectic form $\omega$. In the same way, one can say that the space $V$ chooses the reparametrization group $Q S\left(S^{1}\right)$. Indeed, this is the biggest reparametrization group, leaving $V$ invariant, according to Proposition 1. On the other hand, it is a group of "canonical transformations", preserving the symplectic form $\omega$, according to Proposition 2 . So we have a natural phase space $(V, \omega)$ together with a natural group $Q S\left(S^{1}\right)$ of its canonical transformations.

Here is an assertion, making clear in what sense $\omega$ is a unique natural symplectic form on $V$.

Proposition 3 (Nag-Sullivan [12]). Suppose that $\tilde{\omega}: V \times V \rightarrow \mathbb{R}$ is a continuous bilinear form on $V$ such that

$$
\tilde{\omega}\left(h^{*}(\xi), h^{*}(\eta)\right)=\tilde{\omega}(\xi, \eta)
$$

for all $\xi, \eta \in V$ and all $h \in \operatorname{Möb}\left(S^{1}\right)$. Then $\tilde{\omega}=\lambda \omega$ for some real constant $\lambda$. In particular, $\tilde{\omega}$ is non-degenerate (if it is not identically zero) and invariant under the whole group $\operatorname{QS}\left(S^{1}\right)$.

\subsection{Embedding of the universal Teichmüller space into an infinite-dimensional Siegel disc}

The Propositions 1 and 2 imply that quasisymmetric homeomorphisms act on the Hilbert space $V$ by bounded symplectic operators. Hence, we have a map

$$
\mathcal{T}=\operatorname{QS}\left(S^{1}\right) / \operatorname{Möb}\left(S^{1}\right) \longrightarrow \operatorname{Sp}(V) / \mathrm{U}\left(W_{+}\right) \text {. }
$$

Here, $\operatorname{Sp}(V)$ is the symplectic group of $V$, consisting of linear bounded symplectic operators on $V$, and $\mathrm{U}\left(W_{+}\right)$is its subgroup, consisting of unitary operators (i.e. the operators, whose complex-linear extensions to $V^{\mathbb{C}}$ preserve the subspace $\left.W_{+}\right)$.

In terms of the decomposition

$$
V^{\mathbb{C}}=W_{+} \oplus W_{-}
$$

any linear operator $A: V^{\mathbb{C}} \rightarrow V^{\mathbb{C}}$ is written in the block form

$$
A=\left(\begin{array}{ll}
a & b \\
c & d
\end{array}\right) .
$$

Such an operator belongs to symplectic group $\operatorname{Sp}(V)$, if it has the form

$$
A=\left(\begin{array}{ll}
a & b \\
\bar{b} & \bar{a}
\end{array}\right)
$$

with components, satisfying the relations

$$
\bar{a}^{t} a-b^{t} \bar{b}=1, \quad \bar{a}^{t} b=b^{t} \bar{a},
$$

where $a^{t}, b^{t}$ denote the transposed operators $a^{t}: W_{-} \rightarrow W_{-}, b^{t}: W_{-} \rightarrow W_{+}$. The unitary group $\mathrm{U}\left(W_{+}\right)$is embedded into $\operatorname{Sp}(V)$ as a subgroup, consisting of diagonal block matrices of the form

$$
A=\left(\begin{array}{ll}
a & 0 \\
0 & \bar{a}
\end{array}\right) .
$$

The space

$$
\operatorname{Sp}(V) / \mathrm{U}\left(W_{+}\right),
$$


standing on the right hand side of (6), can be regarded as an infinite-dimensional analogue of the Siegel disc, since it may be identified with the space of complex structures on $V$, compatible with $\omega$. Indeed, any such structure $J$ determines a decomposition

$$
V^{\mathbb{C}}=W \oplus \bar{W}
$$

of $V^{\mathbb{C}}$ into the direct sum of subspaces, isotropic with respect to $\omega$. This decomposition is orthogonal with respect to the Kähler metric $g_{J}$ on $V^{\mathbb{C}}$, determined by $J$ and $\omega$. The subspaces $W$ and $\bar{W}$ are identified with the $(-i)$ - and $(+i)$-eigenspaces of the operator $J$ on $V^{\mathbb{C}}$ respectively. Conversely, any decomposition (7) of the space $V^{\mathbb{C}}$ into the direct sum of isotropic subspaces determines a complex structure $J$ on $V^{\mathbb{C}}$, which is equal to $-i I$ on $W$ and $+i I$ on $\bar{W}$ and is compatible with $\omega$. This argument shows that symplectic group $\operatorname{Sp}(V)$ acts transitively on the space $\mathcal{J}(V)$ of complex structures $J$ on $V$, compatible with $\omega$. Moreover, a complex structure $J$, obtained from a reference complex structure $J^{0}$ by the action of an element $A$ of $\operatorname{Sp}(V)$, is equivalent to $J^{0}$ if and only if $A \in \mathrm{U}\left(W_{+}\right)$. Hence,

$$
\operatorname{Sp}(V) / \mathrm{U}\left(W_{+}\right)=\mathcal{J}(V) .
$$

The space on the right can be, in its turn, identified with the Siegel disc $\mathcal{D}$, defined as the set

$$
\mathcal{D}=\left\{Z: W_{+} \rightarrow W_{-} \text {is a symmetric bounded linear operator with } \bar{Z} Z<I\right\} .
$$

The symmetricity of $Z$ means that $Z^{t}=Z$ and the condition $\bar{Z} Z<I$ means that symmetric operator $I-\bar{Z} Z$ is positive definite. In order to identify $\mathcal{J}(V)$ with $\mathcal{D}$, consider the action of the group $\operatorname{Sp}(V)$ on $\mathcal{D}$, given by fractional-linear transformations $A: \mathcal{D} \rightarrow \mathcal{D}$ of the form

$$
Z \longmapsto(\bar{a} Z+\bar{b})(b Z+a)^{-1}
$$

where $A=\left(\begin{array}{ll}a & b \\ \bar{b} & \bar{a}\end{array}\right) \in \operatorname{Sp}(V)$. The isotropy subgroup at $Z=0$ coincides with the set of operators $A \in \operatorname{Sp}(V)$ such that $b=0$, i.e. with $\mathrm{U}\left(W_{+}\right)$.

So the space

$$
\mathcal{J}(V)=\operatorname{Sp}(V) / \mathrm{U}\left(W_{+}\right)
$$

can be identified with the Siegel disc $\mathcal{D}$, and we have the following

Proposition 4 (Nag-Sullivan [12]). The map

$$
\mathcal{T}=Q S\left(S^{1}\right) / \operatorname{Möb}\left(S^{1}\right) \hookrightarrow \mathcal{J}(V)=\operatorname{Sp}(V) / U\left(W_{+}\right)=\mathcal{D}
$$

is an equivariant holomorphic embedding of Banach manifolds.

For the smooth part $\mathcal{S}$ of the universal Teichmüller space we can obtain a stronger version of this assertion by replacing symplectic group $\operatorname{Sp}(V)$ with its Hilbert-Schmidt subgroup $\operatorname{Sp}_{\mathrm{HS}}(V)$. By definition, this subgroup consists of bounded linear operators $A \in \operatorname{Sp}(V)$ with block representations

$$
A=\left(\begin{array}{ll}
a & b \\
\bar{b} & \bar{a}
\end{array}\right)
$$

in which the operator $b$ is Hilbert-Schmidt. 
The map $f \mapsto T_{f}$ defines an embedding

$$
\mathcal{S} \hookrightarrow \operatorname{Sp}_{\mathrm{HS}}(V) / \mathrm{U}\left(W_{+}\right) .
$$

We identify, as above, the right hand side with a subspace $\mathcal{J}_{\mathrm{HS}}(V)$ of the space $\mathcal{J}(V)$ of compatible complex structures on $V$. We call complex structures $J \in \mathcal{J}_{\mathrm{HS}}(V)$ Hilbert-Schmidt. As before, the space $\mathcal{J}_{\mathrm{HS}}(V)$ of Hilbert-Schmidt complex structures on $V$ can be realized as a Hilbert-Schmidt Siegel disc

$$
\mathcal{D}_{\mathrm{HS}}=\left\{Z: W_{+} \rightarrow W_{-} \text {is a symmetric Hilbert-Schmidt operator with } \bar{Z} Z<I\right\} .
$$

We have

Proposition 5 (Nag [11]). The map

$$
\mathcal{S}=\operatorname{Diff}_{+}\left(S^{1}\right) / \operatorname{Möb}\left(S^{1}\right) \hookrightarrow \mathcal{J}_{\mathrm{HS}}(V)=\operatorname{Sp}_{\mathrm{HS}}(V) / \mathrm{U}\left(W_{+}\right)=\mathcal{D}_{\mathrm{HS}}
$$

is an equivariant holomorphic embedding.

\section{Quantization of $\mathcal{S}$}

\section{Statement of the problem}

\subsection{Dirac quantization}

We start by recalling a general definition of quantization of finite-dimensional classical systems, due to Dirac. A classical system is given by a pair $(M, \mathcal{A})$, where $M$ is the phase space and $\mathcal{A}$ is the algebra of observables.

The phase space $M$ is a smooth symplectic manifold of even dimension $2 n$, provided with a symplectic 2 -form $\omega$. Locally, it is equivalent to the standard model, given by symplectic vector space $M_{0}:=\mathbb{R}^{2 n}$ together with standard symplectic form $\omega_{0}$, given in canonical coordinates $\left(p_{i}, q_{i}\right), i=1, \ldots, n$, on $\mathbb{R}^{2 n}$ by

$$
\omega_{0}=\sum_{i=1}^{n} d p_{i} \wedge d q_{i} .
$$

The algebra of observables $\mathcal{A}$ is a Lie subalgebra of the Lie algebra $C^{\infty}(M, \mathbb{R})$ of smooth real-valued functions on the phase space $M$, provided with the Poisson bracket, determined by symplectic 2 -form $\omega$. In particular, in the case of standard model $M_{0}=\left(\mathbb{R}^{2 n}, \omega_{0}\right)$ one can take for $\mathcal{A}$ the Heisenberg algebra heis $\left(\mathbb{R}^{2 n}\right)$, which is the Lie algebra, generated by coordinate functions $p_{i}, q_{i}, i=1, \ldots, n$, and 1 , satisfying the commutation relations

$$
\begin{aligned}
& \left\{p_{i}, p_{j}\right\}=\left\{q_{i}, q_{j}\right\}=0, \\
& \left\{p_{i}, q_{j}\right\}=\delta_{i j} \quad \text { for } \quad i, j=1, \ldots, n .
\end{aligned}
$$

Definition 3. The Dirac quantization of a classical system $(M, \mathcal{A})$ is an irreducible Lie-algebra representation

$$
r: \mathcal{A} \longrightarrow \text { End }^{*} H
$$

of the algebra of observables $\mathcal{A}$ in the algebra of linear self-adjoint operators, acting on a complex Hilbert space $H$, called the quantization space. The algebra End $^{*} H$ is provided with the Lie 
bracket, given by the commutator of linear operators of the form $\frac{1}{i}[A, B]$. In other words, it is required that

$$
r(\{f, g\})=\frac{1}{i}(r(f) r(g)-r(g) r(f))
$$

for any $f, g \in \mathcal{A}$. We also assume the following normalization condition: $r(1)=I$.

For complexified algebras of observables $\mathcal{A}^{\mathbb{C}}$ or, more generally, complex involutive Lie algebras of observables (i.e. Lie algebras with conjugation) their Dirac quantization is given by an irreducible Lie-algebra representation

$$
r: \mathcal{A}^{\mathbb{C}} \longrightarrow \text { End } H
$$

satisfying the normalization condition and the conjugation law: $r(\bar{f})=r(f)^{*}$ for any $f \in \mathcal{A}$.

We are going to apply this definition of quantization to infinite-dimensional classical systems, in which both the phase space and algebra of observables are infinite-dimensional. For infinitedimensional algebras of observables it is more natural to look for their projective Lie-algebra representations. The above definition of quantization will apply also to this case if one replaces the original algebra of observables with its suitable central extension.

\subsection{Statement of the problem}

We start from the Dirac quantization of an infinite-dimensional system $(V, \mathcal{A})$ with the phase space, given by the Sobolev space of half-differentiable functions $V:=H_{0}^{1 / 2}\left(S^{1}, \mathbb{R}\right)$. The role of algebra of observables $\mathcal{A}$ will be played by the semi-direct product

$$
\mathcal{A}=\operatorname{heis}(V) \rtimes \operatorname{sp}_{\mathrm{HS}}(V),
$$

being the Lie algebra of the Lie group $\mathcal{G}=\operatorname{Heis}(V) \rtimes \operatorname{Sp}_{\mathrm{HS}}(V)$. The symplectic Hilbert-Schmidt group $\operatorname{Sp}_{\mathrm{HS}}(V)$ was introduced in Section 4.2, while the Heisenberg algebra heis $(V)$ and the corresponding Heisenberg group Heis $(V)$ are defined, as in finite-dimensional situation. Namely, the Heisenberg algebra heis $(V)$ of $V$ is a central extension of the Abelian Lie algebra $V$, generated by coordinate functions. In other words, it coincides, as a vector space, with heis $(V)=V \oplus \mathbb{R}$, provided with the Lie bracket

$$
[(x, s),(y, t)]:=(0, \omega(x, y)), \quad x, y \in V, \quad s, t, \in \mathbb{R} .
$$

Respectively, the Heisenberg group $\operatorname{Heis}(V)$ is a central extension of the Abelian group $V$, i.e. the direct product Heis $(V)=V \times S^{1}$, provided with the group operation, given by

$$
(x, \lambda) \cdot(y, \mu):=\left(x+y, \lambda \mu e^{i \omega(x, y)}\right) .
$$

The choice of the introduced Lie algebra $\mathcal{A}$ for the algebra of observables is motivated by the following physical considerations. As we have pointed put, the space $V_{d}$ is a natural Sobolev completion of the space $\Omega_{d}:=C_{0}^{\infty}\left(S^{1}, \mathbb{R}^{d}\right)$ of smooth loops in $\mathbb{R}^{d}$. In the same way, the Lie algebra $\mathcal{A}=\operatorname{heis}(V) \rtimes \operatorname{sp}_{\mathrm{HS}}(V)$ is a natural extension of the Lie algebra heis $\left(\Omega_{d}\right) \rtimes \operatorname{Vect}\left(S^{1}\right)$, where $\operatorname{Vect}\left(S^{1}\right)$ is the Lie algebra of the diffeomorphism group $\operatorname{Diff}_{+}\left(S^{1}\right)$. The algebra heis $\left(\Omega_{d}\right)$ can be identified with the Lie algebra of coordinate functions on $\Omega_{d}$, while the algebra $\operatorname{Vect}\left(S^{1}\right)$ is generated by certain quadratic functions on $\Omega_{d}$ (cf. [3]). One can say that the Lie algebra heis $\left(\Omega_{d}\right) \rtimes \operatorname{Vect}\left(S^{1}\right)$ is an infinite-dimensional analogue of the Poincarè algebra of the $d$ dimensional Minkowski space $M^{d}$, where heis $\left(\Omega_{d}\right)$ plays the role of the Lie algebra of translations of $M^{d}$, while $\operatorname{Vect}\left(S^{1}\right)$ is an analogue of the Lie algebra of hyperbolic rotations of $M^{d}$. 


\section{$7 \quad$ Heisenberg representation}

In this Section we recall the well known Heisenberg representation of the first component heis $(V)$ of algebra of observables $\mathcal{A}$. A detailed exposition of this subject may be found in $[13,8,2]$.

\section{$7.1 \quad$ Fock space}

Fix an admissible complex structure $J \in \mathcal{J}(V)$. It defines a polarization of $V$, i.e. a decomposition of $V^{\mathbb{C}}$ into the direct sum

$$
V^{\mathbb{C}}=W \oplus \bar{W},
$$

where $W$ (resp. $\bar{W})$ is the $(-i)$-eigenspace (resp. $(+i)$-eigenspace) of the complex structure operator $J$. The splitting (8) is the orthogonal direct sum with respect to the Hermitian inner product $\langle z, w\rangle_{J}:=\omega(z, J w)$, determined by $J$ and sympletic form $\omega$.

The Fock space $F\left(V^{\mathbb{C}}, J\right)$ is the completion of the algebra of symmetric polynomials on $W$ with respect to a natural norm, generated by $\langle\cdot, \cdot\rangle_{J}$. In more detail, denote by $S(W)$ the algebra of symmetric polynomials in variables $z \in W$ and introduce an inner product on $S(W)$, defined in the following way. It is given on monomials of the same degree by the formula

$$
\left\langle z_{1} \cdots z_{n}, z_{1}^{\prime} \cdots z_{n}^{\prime}\right\rangle_{J}=\sum_{\left\{i_{1}, \ldots, i_{n}\right\}}\left\langle z_{1}, z_{i_{1}}^{\prime}\right\rangle_{J} \cdots\left\langle z_{n}, z_{i_{n}}^{\prime}\right\rangle_{J}
$$

where the summation is taken over all permutations $\left\{i_{1}, \ldots, i_{n}\right\}$ of the set $\{1, \ldots, n\}$ (the inner product of monomials of different degrees is set to zero), and extended to the whole algebra $S(W)$

by linearity. The completion $\widehat{S(W)}$ of $S(W)$ with respect to the introduced norm is called the Fock space of $V^{\mathbb{C}}$ with respect to complex structure $J$ :

$$
F_{J}=F\left(V^{\mathbb{C}}, J\right):=\widehat{S(W)} .
$$

If $\left\{w_{n}\right\}, n=1,2, \ldots$, is an orthonormal basis of $W$, then an orthonormal basis of $F_{J}$ can be given by the family of polynomials

$$
P_{K}(z)=\frac{1}{\sqrt{k !}}\left\langle z, w_{1}\right\rangle_{J}^{k_{1}} \cdots\left\langle z, w_{n}\right\rangle_{J}^{k_{n}}, \quad z \in W
$$

where $K=\left(k_{1}, \ldots, k_{n}, 0, \ldots\right), k_{i} \in \mathbb{N} \cup 0$, and $k !=k_{1} ! \cdots k_{n} !$.

\subsection{Heisenberg representation}

There is an irreducible representation of the Heisenberg algebra heis $(V)$ in the Fock space $F_{J}=F\left(V^{\mathbb{C}}, J\right)$, defined in the following way. Elements of $S(W)$ may be considered as holomorphic functions on $\bar{W}$, if we identify $z \in W$ with a holomorphic function $\bar{w} \mapsto\langle w, z\rangle$ on $\bar{W}$. Accordingly, $F_{J}$ may be considered as a subspace of the space $\mathcal{O}(\bar{W})$ of functions, holomorphic on $\bar{W}$. With this convention the Heisenberg representation

$$
r_{J}: \operatorname{heis}(V) \longrightarrow \operatorname{End}^{*} F_{J}
$$

of the Heisenberg algebra heis $(V)$ in the Fock space $F_{J}=F\left(V^{\mathbb{C}}, J\right)$ is defined by the formula

$$
r_{J}(v) f(\bar{w}):=-\partial_{v} f(\bar{w})+\langle w, v\rangle_{J} f(\bar{w}),
$$

where $\partial_{v}$ is the derivative in direction of $v \in V$. Extending $r_{J}$ to the complexified algebra heis ${ }^{\mathbb{C}}(V)$, we obtain

$$
r_{J}(\bar{z}) f(\bar{w}):=-\partial_{\bar{z}} f(\bar{w})
$$


for $v=\bar{z} \in \bar{W}$ and

$$
r_{J}(z) f(\bar{w}):=\langle w, z\rangle_{J} f(\bar{w})
$$

for $z \in W$. We set also $r_{J}(c):=\lambda \cdot I$ for the central element $c \in$ heis $(V)$, where $\lambda$ is an arbitrary fixed non-zero constant.

Introduce the creation and annihilation operators on $F_{J}$, defined for $v \in V^{\mathbb{C}}$ by

$$
a_{J}^{*}(v):=\frac{r_{J}(v)-i r_{J}(J v)}{2}, \quad a_{J}(v):=\frac{r_{J}(v)+i r_{J}(J v)}{2} .
$$

In particular, for $z \in W$

$$
a_{J}^{*}(z) f(\bar{w})=\langle w, z\rangle_{J} f(\bar{w}),
$$

and for $\bar{z} \in \bar{W}$

$$
a_{J}(\bar{z}) f(\bar{w})=-\partial_{\bar{z}} f(\bar{w}) .
$$

For an orthonormal basis $\left\{w_{n}\right\}$ of $W$, we define the operators

$$
a_{n}^{*}:=a^{*}\left(w_{n}\right), \quad a_{n}:=a\left(\bar{w}_{n}\right), \quad n=1,2, \ldots,
$$

and $a_{0}:=\lambda \cdot I$.

A vector $f_{J} \in F_{J} \backslash\{0\}$ is called the vacuum, if $a_{n} f_{J}=0$ for $n=1,2, \ldots$ In other words, it is a non-zero vector, annihilated by operators $a_{n}$. It is uniquely defined by $r_{J}$ (up to a multiplicative constant) and in the case of the initial Fock space $F_{0}=F\left(V, J^{0}\right)$ we set $f_{0} \equiv 1$. Acting on vacuum $f_{J}$ by creation operators $a_{n}^{*}$, we can define the action of representation $r_{J}$ on any polynomial, which implies the irreducubility of $r_{J}$.

So we have the following

Proposition 6 (cf. $[\mathbf{1 3}, \mathbf{8 ,}$ 2]). There is an irredicible Lie algebra representation

$$
r_{J}: \operatorname{heis}(V) \longrightarrow \text { End }^{*} F_{J}
$$

of the Heisenberg algebra heis $(V)$ in the Fock space $F_{J}=F\left(V^{\mathbb{C}}, J\right)$, given by the formula (10).

We shall see in the next Section that this representation is essentially unique.

\section{Symplectic group action on the Fock bundle}

\subsection{Shale theorem}

To construct an irreducible representation of the second component $\operatorname{sp}_{\mathrm{HS}}(V)$ of the algebra of observables $\mathcal{A}$, we study an action of the Hilbert-Schmidt symplectic group $\operatorname{Sp}_{\mathrm{HS}}(V)$ on the Fock spaces $F_{J}$. This action is provided by the following theorem.

Theorem 3 (Shale). The representations $r_{0}$ in $F_{0}$ and $r_{J}$ in $F_{J}$ are unitary equivalent if and only if $J \in \mathcal{J}_{\mathrm{HS}}(V)$. In other words, for $J \in \mathcal{J}_{\mathrm{HS}}(V)$ there exists a unitary intertwining operator $U_{J}: F_{0} \rightarrow F_{J}$ such that

$$
r_{J}(v)=U_{J} \circ r_{0}(v) \circ U_{J}^{-1} .
$$

This theorem was proved by Shale [17] in 1962, an independent proof was given in Berezin's book [2], published in Russian in 1965 (Berezin obtained also an explicit formula for the intertwining operator $U_{J}$ ).

The following Proposition gives a description of $U_{J}$ in terms of the Hilbert-Schmidt Siegel disc $\mathcal{D}_{\mathrm{HS}}$, based on the identification of $\mathcal{J}_{\mathrm{HS}}(V)$ with $\mathcal{D}_{\mathrm{HS}}$. 
Proposition 7 (Segal [16]). There is a projective unitary action of the group $\operatorname{Sp}_{\mathrm{HS}}(V)$ on Fock spaces, defined by the unitary operator $U_{J}$, given by the formula (11) below.

Here is an idea of Segal's construction, details may be found in [16]. Given an admissible complex structure $J \in \mathcal{J}_{\mathrm{HS}}(V)$, we identify it with a point $Z$ in the Siegel disc $\mathcal{D}_{\mathrm{HS}}$. Regarding $Z$ as an element of the symmetric square $\widehat{S}^{2}(W)$, we can associate with it an element $e^{Z / 2}$ of $\widehat{S(W)}$. The inner product of two such elements has a simple expression

$$
\left\langle e^{Z_{1} / 2}, e^{Z_{2} / 2}\right\rangle=\operatorname{det}\left(1-\bar{Z}_{1} Z_{2}\right)^{-1 / 2}
$$

The normalized elements

$$
\epsilon_{Z}:=\operatorname{det}(1-\bar{Z} Z)^{1 / 4} e^{Z / 2}
$$

play the role of coherent states (cf., e.g., [2]). In terms of these states the action of the group $\operatorname{Sp}_{\mathrm{HS}}(V)$ on Fock spaces, defined by

$$
\operatorname{Sp}_{\mathrm{HS}}(V) \ni A=\left(\begin{array}{cc}
a & b \\
\bar{b} & \bar{a}
\end{array}\right) \longmapsto U_{J}: F_{0} \rightarrow F_{J} \quad \text { for } \quad J=A \cdot J^{0}
$$

is given by the formula

$$
U_{J}: \epsilon_{Z} \longmapsto \mu \operatorname{det}\left(1+a^{-1} \bar{b} Z\right)^{1 / 2} \epsilon_{A \cdot Z},
$$

where $\mu: \mathbb{C}^{*} \rightarrow S^{1}$ is the radial projection.

\subsection{Dirac quantization of $V$ and $\mathcal{S}$}

We can unite Fock spaces $F_{J}$ into a Fock bundle over $\mathcal{D}_{\mathrm{HS}}$, having the following properties.

Proposition 8. The Fock bundle

$$
\mathcal{F}:=\bigcup_{J \in \mathcal{J}(V)} F_{J} \longrightarrow \mathcal{J}(V)=\mathcal{D}_{\mathrm{HS}}
$$

is a Hermitian holomorphic Hilbert space bundle over $\mathcal{D}_{\mathrm{HS}}$. It can be provided with a projective unitary action of the group $\operatorname{Sp}_{\mathrm{HS}}(V)$, covering the natural $\operatorname{Sp}_{\mathrm{HS}}(V)$-action on the Siegel disc $\mathcal{D}_{\mathrm{HS}}$.

The proof of holomorphicity of the Fock bundle $\mathcal{F} \rightarrow \mathcal{D}_{\mathrm{HS}}$ is analogous to the proof of holomorphicity of the determinant bundle over the Hilbert-Schmidt Grassmannian, given in [13]. Note that the Fock bundle is trivial, since the Siegel disc $\mathcal{D}_{\text {HS }}$ is contractible (even convex), so the statement follows from the Hilbert space version of the Oka principle (cf. [4]). An explicit trivialization of $\mathcal{F} \rightarrow \mathcal{D}_{\mathrm{HS}}$ is provided by the action (11). This action defines a projective unitary action of the group $\operatorname{Sp}_{\mathrm{HS}}(V)$ on $\mathcal{F}$, covering the $\operatorname{Sp}_{\mathrm{HS}}(V)$-action on Siegel disc $\mathcal{D}_{\mathrm{HS}}$.

The infinitesimal version of this action yields a projective representation of the symplectic algebra $\operatorname{sp}_{\mathrm{HS}}(V)$ in the Fock space $F_{0}$. We present an explicit description of this representation, due to Segal.

Recall that symplectic algebra $\operatorname{sp}_{\mathrm{HS}}(V)$ is the Lie algebra of symplectic Hilbert-Schmidt group $\operatorname{Sp}_{\mathrm{HS}}(V)$, which consists of linear operators $A$ in $V^{\mathbb{C}}$, having the following block representations

$$
A=\left(\begin{array}{cc}
\alpha & \beta \\
\bar{\beta} & \bar{\alpha}
\end{array}\right) .
$$


Here, $\alpha$ is a bounded skew-Hermitian operator and $\beta$ is a symmetric Hilbert-Schmidt operator on $F_{0}$. The complexified Lie algebra $\operatorname{sp}_{\mathrm{HS}}(V)^{\mathbb{C}}$ consists of operators of the form

$$
A=\left(\begin{array}{cc}
\alpha & \beta \\
\bar{\gamma} & -\alpha^{t}
\end{array}\right),
$$

where $\alpha$ is a bounded operator, while $\beta$ and $\bar{\gamma}$ are symmetric Hilbert-Schmidt operators on $F_{0}$.

The projective representation of complexified symplectic algebra $\operatorname{sp}_{\mathrm{HS}}(V)^{\mathbb{C}}$ is given by the formula

$$
\operatorname{sp}_{\mathrm{HS}}(V)^{\mathbb{C}} \ni A=\left(\begin{array}{cc}
\alpha & \beta \\
\bar{\gamma} & -\alpha^{t}
\end{array}\right) \longmapsto \rho(A)=D_{\alpha}+\frac{1}{2} M_{\beta}+\frac{1}{2} M_{\gamma}^{*} .
$$

Here, $D_{\alpha}$ is the derivation of $F_{0}$ in $\alpha$-direction, defined by

$$
D_{\alpha} f(\bar{w})=\left\langle\alpha w, \partial_{\bar{w}}\right\rangle f(\bar{w}) .
$$

The operator $M_{\beta}$ is the multiplication operator on $F_{0}$, defined by

$$
M_{\beta} f(\bar{w})=\langle\bar{\beta} w, \bar{w}\rangle f(\bar{w}),
$$

and the operator $M_{\gamma}^{*}$ is the adjoint of $M_{\gamma}: M_{\gamma}^{*} f(\bar{w})=\left\langle\gamma \partial_{w}, \partial_{\bar{w}}\right\rangle f(\bar{w})$.

This is a projective representation with cocycle

$$
\left[\rho\left(A_{1}\right), \rho\left(A_{2}\right)\right]-\rho\left(\left[A_{1}, A_{2}\right]\right)=\frac{1}{2} \operatorname{tr}\left(\bar{\gamma}_{2} \beta_{1}-\bar{\gamma}_{1} \beta_{2}\right) I,
$$

intertwined with the Heisenberg representation $r_{0}$ of heis $(V)$ in $F_{0}$.

Thus we have the following

Proposition 9 (Segal [16]). There is a projective unitary representation

$$
\rho: \operatorname{sp}_{\mathrm{HS}}(V) \longrightarrow \text { End }^{*} F_{0},
$$

given by formula (12) with cocycle (13). This representation intertwines with the Heisenberg representation $r_{0}$ of heis $(V)$ in $F_{0}$.

The Heisenberg representation $r_{0}$ in the Fock space $F_{0}$, described in Proposition 6 , and symplectic representation $\rho$, constructed in Proposition 9, define together Dirac quantization of the system $(V, \widetilde{\mathcal{A}})$, where $\widetilde{\mathcal{A}}$ is the central extension of $\mathcal{A}$, determined by (13).

The constructed Lie-algebra representation of $\operatorname{sp}_{\mathrm{HS}}(V)$ in the Fock space $F_{0}$ may be also considered as Dirac quantization of a classical system, consisting of the phase space $\mathcal{D}_{\mathrm{HS}}=$ $\operatorname{Sp}_{\mathrm{HS}}(V) / \mathrm{U}\left(W_{+}\right)$and the algebra of observables, given by the central extension of Lie algebra $\operatorname{sp}_{\mathrm{HS}}(V)$.

The restriction of this construction to the smooth part $\mathcal{S}=\operatorname{Diff}_{+}\left(S^{1}\right) / \operatorname{Möb}\left(S^{1}\right)$ of the universal Teichmüller space $\mathcal{T}=\operatorname{QS}\left(S^{1}\right) / \operatorname{Möb}\left(S^{1}\right)$ yields the Dirac quantization of $\mathcal{S}$. Namely, we have the following

Proposition 10. The restriction of the Fock bundle $\mathcal{F} \rightarrow \mathcal{D}_{\mathrm{HS}}$ to $\mathcal{S}$ is a Hermitian holomorphic Hilbert space bundle

$$
\mathcal{F}:=\bigcup_{J \in \mathcal{S}} F_{J} \longrightarrow \mathcal{S}
$$

over $\mathcal{S}$. This bundle is provided with a projective unitary action of the diffeomorphism group $\operatorname{Diff}_{+}\left(S^{1}\right)$, covering the natural $\operatorname{Diff}_{+}\left(S^{1}\right)$-action on $\mathcal{S}$.

The $\operatorname{Diff}_{+}\left(S^{1}\right)$-action on the Fock bundle, mentioned in Proposition, was explicitly constructed in [7]. The infinitesimal version of this action yields a unitary projective representation of the Lie algebra $\operatorname{Vect}\left(S^{1}\right)$ in the Fock space $F_{0}$. We can consider this construction as Dirac quantization of the phase space $\mathcal{S}$, provided with the algebra of observables, given by the central extension of the Lie algebra $\operatorname{Vect}\left(S^{1}\right)$, called the Virasoro algebra. 


\section{Quantization of $\mathcal{T}$}

\section{Dirac versus Connes quantization}

Unfortunately, the method, used in previous Chapter for the quantization of $\mathcal{S}$, does not apply to the whole space $\mathcal{T}$. Though we still can embed $\mathcal{T}$ into the Siegel disc $\mathcal{D}$, we are not able to construct a projective action of symplectic group $\operatorname{Sp}(V)$ on Fock spaces. According to theorem of Shale, it is possible only for the Hilbert-Schmidt subgroup $\operatorname{Sp}_{\mathrm{HS}}(V)$ of $\operatorname{Sp}(V)$. So one should look for another way of quantizing the universal Teichmüller space $\mathcal{T}$. We are going to use for that the "quantized calculus" of Connes and Sullivan, presented in Chapter IV of the Connes' book [5] and [12].

Recall that in Dirac's approach we quantize a classical system $(M, \mathcal{A})$, consisting of the phase space $M$ and the algebra of observables $\mathcal{A}$, which is a Lie algebra, consisting of smooth functions on $M$. The quantization of this system is given by a representation $r$ of $\mathcal{A}$ in a Hilbert space $H$, sending the Poisson bracket $\{f, g\}$ of functions $f, g \in \mathcal{A}$ into the commutator $\frac{1}{i}[r(f), r(g)]$ of the corresponding operators. In Connes' approach the algebra of observables $\mathfrak{A}$ is an associative involutive algebra, provided with an exterior differential $d$. Its quantization is, by definition, a representation $\pi$ of $\mathfrak{A}$ in a Hilbert space $H$, sending the differential $d f$ of a function $f \in \mathfrak{A}$ into the commutator $[S, \pi(f)]$ of the operator $\pi(f)$ with a self-adjoint symmetry operator $S$ with $S^{2}=I$. The differential here is understood in the sense of non-commutative geometry, i.e. as a linear map $d: \mathfrak{A} \rightarrow \Omega^{1}(\mathfrak{A})$, satisfying the Leibnitz rule (cf. [5]).

In the following table we compare Connes and Dirac approaches to quantization.

\begin{tabular}{|c|c|c|}
\hline & Dirac approach & Connes approach \\
\hline 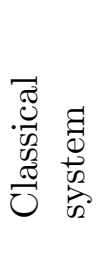 & $\begin{array}{c}(M, \mathcal{A}) \text { where: } \\
M-\text { phase space } \\
\mathcal{A}-\text { involutive Lie algebra } \\
\text { of observables }\end{array}$ & $\begin{array}{c}(M, \mathfrak{A}) \text { where: } \\
M-\text { phase space } \\
\mathfrak{A}-\text { involutive associative } \\
\text { algebra of observables with } \\
\text { differential } d: \mathfrak{A} \rightarrow \Omega^{1}(\mathfrak{A})\end{array}$ \\
\hline 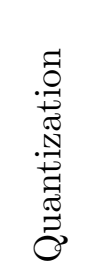 & $\begin{array}{c}\text { Lie-algebra representation } \\
\qquad \begin{aligned} r: \mathcal{A} & \rightarrow \text { End } H, \\
& \text { sending } \\
\{f, g\} & \mapsto \frac{1}{i}[r(f), r(g)]\end{aligned}\end{array}$ & $\begin{array}{c}\text { representation } \\
\pi: \mathfrak{A} \rightarrow \text { End } H, \\
\text { sending } \\
\quad d f \mapsto[S, \pi(f)], \\
\text { where } S=S^{*}, S^{2}=I\end{array}$ \\
\hline
\end{tabular}

Reformulating the notion of Connes quantization of algebra of observables $\mathfrak{A}$, one can say that it is a representation of the algebra $\operatorname{Der}(\mathfrak{A})$ of derivations of $\mathfrak{A}$ in the Lie algebra End $H$. Recall that a derivation of an algebra $\mathfrak{A}$ is a linear map: $\mathfrak{A} \rightarrow \mathfrak{A}$, satisfying the Leibnitz rule. Clearly, derivations of an algebra $\mathfrak{A}$ form a Lie algebra, since the commutator of two derivations is again a derivation.

If all observables are smooth real-valued functions on $M$, the two approaches are equivalent to each other. Indeed, the differential $d f$ of a smooth function $f$ is symplectically dual to the Hamiltonian vector field $X_{f}$ and this establishes a relation between the associative algebra $\mathfrak{A}$ of functions $f$ on $M$ and the Lie algebra $\mathcal{A}$ of Hamiltonian vector fields on $M$. (This Lie algebra is isomorphic for a simply connected $M$ to a Lie algebra of Hamiltonians, associated with $\mathcal{A}$.) A symmetry operator $S$ is determined by a polarization $H=H_{+} \oplus H_{-}$of the quantization space $H$. Evidently, $S=i J$, where $J$ is the complex structure operator, defining the polarization $H=H_{+} \oplus H_{-}$. (By this reason we do not make distinction between symmetry and complex structure operators.) 
In the case when the algebra of observables $\mathcal{A}$ contains non-smooth functions, its Dirac quantization is not defined in the classical sense. In Connes approach the differential $d f$ of a non-smooth observable $f \in \mathfrak{A}$ is also not defined classically, but its quantum counterpart $d^{q} f$, given by

$$
d^{q} f:=[S, \pi(f)]
$$

may still be defined, as it is demonstrated by the following example, borrowed from [5].

Suppose that $\mathfrak{A}$ is the algebra $L^{\infty}\left(S^{1}, \mathbb{C}\right)$ of bounded functions on the circle $S^{1}$. Any function $f \in \mathfrak{A}$ defines a bounded multiplication operator in the Hilbert space $H=L^{2}\left(S^{1}, \mathbb{C}\right)$ :

$$
M_{f}: v \in H \longmapsto f v \in H .
$$

The operator $S$ is given by the Hilbert transform $S: L^{2}\left(S^{1}, \mathbb{C}\right) \rightarrow L^{2}\left(S^{1}, \mathbb{C}\right)$ :

$$
(S f)\left(e^{i \varphi}\right)=\frac{1}{2 \pi} V . P . \int_{0}^{2 \pi} K(\varphi, \psi) f\left(e^{i \psi}\right) d \psi,
$$

where the integral is taken in the principal value sense and $K(\varphi, \psi)$ is the Hilbert kernel

$$
K(\varphi, \psi)=1+i \cot \frac{\varphi-\psi}{2} .
$$

The differential $d f$ of a general observable $f \in \mathfrak{A}$ is not defined in the classical sense, but its quantum analogue

$$
d^{q} f:=\left[S, M_{f}\right]
$$

is correctly defined as an operator in $H$ for functions $f \in V$. Namely, we have the following

Proposition 11 (Nag-Sullivan [12]). A function $f \in V$ if and only if the corresponding quantum differential $d^{q} f$ is a Hilbert-Schmidt operator on $H$ (and on $V$ ). Moreover, the HilbertSchmidt norm of $d^{q} f$ coincides with the $V$-norm of $f$.

Indeed, the commutator $d^{q} f:=\left[S, M_{f}\right]$ is an integral operator on $H$ with the kernel, given by $K(\varphi, \psi)(f(\varphi)-f(\psi))$. This operator is Hilbert-Schmidt if and only if its kernel is square integrable on $S^{1} \times S^{1}$, i.e.

$$
\int_{0}^{2 \pi} \int_{0}^{2 \pi} \frac{|f(\varphi)-f(\psi)|^{2}}{\sin ^{2} \frac{1}{2}(\varphi-\psi)} d \varphi d \psi<\infty .
$$

This inequality is equivalent to the condition $f \in V$ (cf. [12]).

The quantum differential $d^{q} f=\left[S, M_{f}\right]$ of a function $f \in V$ is an integral operator on $V$, given by

$$
d^{q} f(h)\left(e^{i \varphi}\right)=\frac{1}{2 \pi} \int_{0}^{2 \pi} k(\varphi, \psi) h\left(e^{i \psi}\right) d \psi
$$

with the kernel, given by

$$
k(\varphi, \psi):=K(\varphi, \psi)(f(\varphi)-f(\psi)),
$$

where $K(\varphi, \psi)$ is defined by (14).

Note that the quasiclassical limit of this operator, defined by taking the value of the kernel on the diagonal (i.e. by taking the limit for $s \rightarrow t$ ), coincides (up to a constant) with the multiplication operator $h \mapsto f^{\prime} h$, so the quantization means in this case essentially the replacement 
of the derivative by its finite-difference analogue. This finite-difference analogue is an integral operator, given by

$$
\delta f(v)\left(e^{i \varphi}\right)=\frac{1}{2 \pi} \int_{0}^{2 \pi} \frac{f(\varphi)-f(\psi)}{\varphi-\psi} v\left(e^{i \psi}\right) d \psi .
$$

The correspondence between functions $f \in \mathfrak{A}$ and operators $M_{f}$ on $H$ has the following remarkable properties (cf. [14]):

1. The differential $d^{q} f$ is a finite rank operator if and only if $f$ is a rational function.

2. The differential $d^{q} f$ is a compact operator if and only if the function $f$ belongs to the class $\operatorname{VMO}\left(S^{1}\right)$.

3. The differential $d^{q} f$ is a bounded operator if and only if the function $f$ belongs to the class $\operatorname{BMO}\left(S^{1}\right)$.

This list may be supplemented by further function-theoretic properties of elements of $\mathfrak{A}$, having curious operator-theoretic characterizations (cf. [5]).

\section{Quantization of the universal Teichmüller space}

We apply these ideas to the universal Teichmüller space $\mathcal{T}$. In Section 5.1 we have defined a natural action of quasisymmetric homeomorphisms on $V$. As we have remarked, this action does not admit the differentiation, so classically there is no Lie algebra, associated with $\operatorname{QS}\left(S^{1}\right)$ or, in other words, there is no classical algebra of observables, associated to $\mathcal{T}$. (The situation is similar to that in the example above.) We would like to define a quantum algebra of observables, associated to $\mathcal{T}$.

First of all, extend the $\operatorname{QS}\left(S^{1}\right)$-action on $V$ to symmetry operators by setting

$$
S^{h}:=h \circ S \circ h^{-1}
$$

for $h \in \mathrm{QS}\left(S^{1}\right)$. This action agrees with a natural action of $\mathrm{QS}\left(S^{1}\right)$ on the universal Teichmüller space $\mathcal{T}=\operatorname{QS}\left(S^{1}\right) / \operatorname{Möb}\left(S^{1}\right)$, considered as a space of compatible complex structures on $V$. The quantized infinitesimal version of the action (16) is given by the integral operator $d^{q} h: V \rightarrow V$, equal to $d^{q} h=[S, \delta h]$ with $\delta h$ given by $(15)$.

Let us recall the steps of the Dirac quantization of Sobolev space $V$ :

1) we start from $\operatorname{Sp}_{\mathrm{HS}}(V)$-action on $V$;

2) extend it to $\operatorname{Sp}_{\mathrm{HS}}(V)$-action on complex structure operators $J$;

3 ) this action generates a projective unitary action of $\operatorname{Sp}_{\mathrm{HS}}(V)$ on Fock spaces $F(V, J)$;

4) the infinitesimal version of this action yields a projective unitary representation of the Lie algebra $\operatorname{sp}_{\mathrm{HS}}(V)$ in Fock space $F_{0}$, described in Section 8.2.

In the case of $\mathcal{T}$ we have:

1) $\operatorname{QS}\left(S^{1}\right)$-action on $V$;

2) this action extends to $\mathrm{QS}\left(S^{1}\right)$-action on symmetry operators $S$, given by $h \mapsto S^{h}$.

However, compared to Dirac quantization of $V$, the next step in the quantization scheme is absent. Because of the Shale theorem, we cannot extend the $\operatorname{QS}\left(S^{1}\right)$-action on symmetry operators $S$ to Fock spaces $F(V, S)$. Also we cannot differentiate the $\operatorname{QS}\left(S^{1}\right)$-action on $V$. But we have a quantized infinitesimal version of $h: S \mapsto S^{h}$, given by quantum differential $d^{q} h=[S, \delta h]$. 
We extend this operator to $F_{0}$ by defining it first on the basis elements $(9)$ of the Fock space $F_{0}$ with the help of Leibnitz rule, and then by linearity to all finite elements of $F_{0}$. The completion of the obtained operator yields an operator $d^{q} h$ on $F_{0}$. These extended operators $d^{q} h$ with $h \in \mathrm{QS}\left(S^{1}\right)$ generate a quantum derivation algebra $\operatorname{Der}^{q}(\mathrm{QS})$, associated to $\mathcal{T}$. This algebra should be considered as a quantum Lie algebra of observables, associated to $\mathcal{T}$. So, instead of steps (3), (4) in the Dirac quantization of $V$, we construct directly a quantum Lie algebra of observables $\operatorname{Der}^{q}(\mathrm{QS})$, corresponding to the non-existing classical Lie algebra of observables on $\mathcal{T}$.

Moreover, we can use the quantum Lie algebra $\operatorname{Der}^{q}(\mathrm{QS})$ as a substitution of a classical Lie algebra of $\mathrm{QS}\left(S^{1}\right)$.

Conclusion. The Connes quantization of the universal Teichmüller space $\mathcal{T}$ consists of two stages:

1. The first stage ("first quantization") is a construction of quantized infinitesimal version of $\mathrm{QS}\left(S^{1}\right)$-action on $V$, given by quantum differentials $d^{q} h=[S, \delta h]$ with $h \in \mathrm{QS}\left(S^{1}\right)$.

2. The second step ("second quantization") is an extension of quantum differentials $d^{q} h$ to the Fock space $F_{0}$. The extended operators $d^{q} h$ with $h \in \mathrm{QS}\left(S^{1}\right)$ generate the quantum algebra of observables $\operatorname{Der}^{q}(\mathrm{QS})$, associated to $\mathcal{T}$.

We note also that the correspondence principle for the constructed Connes quantization of $\mathcal{T}$ means that this quantization reduces to the Dirac quantization while restricted to $\mathcal{S}$.

\section{Acknowledgements}

While preparing this paper, the author was partly supported by the RFBR grants 06-02-04012, 08-01-00014, by the program of Support of Scientific Schools (grant NSH-3224.2008.1), and by the Scientific Program of RAS "Nonlinear Dynamics".

\section{References}

[1] Ahlfors L., Lectures on quaiconformal mappings, Van Nostrand Mathematical Studies, No. 10, D. Van Nostrand Co., Inc., Toronto - New York - London, 1966.

[2] Berezin F.A., Method of second quantization, Pure and Applied Physics, Vol. 24, Academic Press, New York - London, 1966.

[3] Bowick M.J., Rajeev S.G., The holomorphic geometry of closed bosonic string theory and Diff $S^{1} / S^{1}, N u c l e a r$ Phys. B 293 (1987), 348-384.

[4] Bungart L., On analytic fiber bundles. I. Holomorphic fiber bundles with infinite-dimensional fibers, Topology 7 (1967), 55-68.

[5] Connes A., Géométrie non commutative, InterEditions, Paris, 1990.

[6] Douady A., Earle C.J., Conformally natural extension of homeomorphisms of the circle, Acta Math. 157 (1986), 23-48.

[7] Goodman R., Wallach N.R., Projective unitary positive-energy representations of $\operatorname{Diff}\left(S^{1}\right)$, J. Funct. Anal. 63 (1985), 299-321.

[8] Kac V.G., Raina A.K., Highest weight representations of infinite-dimensional Lie algebras, Advanced Series in Mathematical Physics, Vol. 2, World Scientific Publishing Co., Inc., Teaneck, NJ, 1987.

[9] Lehto O., Univalent functions and Teichmüller spaces, Graduate Texts in Mathematics, Vol. 109, SpringerVerlag, New York, 1987.

[10] Nag S., The complex analytic theory of Teichmüller spaces, Canadian Mathematical Society Series of Monographs and Advanced Texts, A Wiley-Interscience Publication, John Wiley \& Sons, Inc., New York, 1988.

[11] Nag S., A period mapping in universal Teichmüller space, Bull. Amer. Math. Soc. (N.S.) 26 (1992), 280-287, math.CA/9204237. 
[12] Nag S., Sullivan D., Teichmüller theory and the universal period mapping via quantum calculus and the $H^{1 / 2}$ space on the circle, Osaka J. Math. 32 (1995), 1-34, alg-geom/9310005.

[13] Pressley A., Segal G., Loop groups, Oxford Mathematical Monographs. Oxford Science Publications, The Clarendon Press, Oxford University Press, New York, 1986.

[14] Power S., Hankel operators on Hilbert space, Research Notes in Mathematics, Vol. 64, Pitman (Advanced Publishing Program), Boston - London, 1982.

[15] Scherk J., An introduction to the theory ofdual models and strings, Rev. Modern Phys. 47 (1975), $123-164$.

[16] Segal G., Unitary representations of some infinite-dimensional groups, Comm. Math. Phys. 80 (1981), 301342.

[17] Shale D., Linear symmetries of free boson field, Trans. Amer. Math. Soc. 103 (1962), 149-167.

[18] Zygmund A., Trigonometric series, Vol. I, II, 3rd ed., Cambridge University Press, Cambridge, 2002. 